\title{
Analysis of Energy Consumption Model and Energy Consumption Characteristics of Electric Vehicle
}

\author{
Taihua Jia \\ Department of Mechanical and Electrical Engineering, Guangdong University of Science \& \\ Technology, Dongguan, China \\ jthdy@foxmail.com
}

\begin{abstract}
Keywords: Energy consumption model; Energy consumption characteristics; Electric vehicle
\end{abstract}
\begin{abstract}
The modeling of energy consumption of electric vehicles is of great significance for meticulous depiction and evaluation of energy saving driving control effect of electric vehicles. Compared with the mesoscopic energy consumption model based on the driving segment, the proposed model can be more accurately estimated. The energy consumption of electric vehicles is generally suitable for detailed characterization of the energy consumption characteristics of electric vehicles, as well as the quantitative estimation of the energy consumption of electric vehicles in small areas such as roads and intersections. In this paper, the driving data of electric vehicle is acquired by the actual driving mode of outdoor roads. The energy consumption model of electric vehicle is established based on the analysis data. Finally, various driving control modes and their energy efficiency improvement are analyzed and evaluated.
\end{abstract}

\section{Introduction}

With global warming, energy crisis and air pollution in the city is increasing, energy saving and emission reduction situation facing traffic field is becoming more and more serious. The development of city low carbon transportation system with low energy consumption, low emission and low pollution characteristics has become the inevitable choice to realize the sustainable development of the city traffic[1-2]. In the process of using zero pollution, low noise and high energy efficiency, the role of electric vehicles in the construction of low carbon traffic is expected in the cities of various countries.

Electric vehicles do not emit harmful gases that pollute the atmosphere, even if they are converted to power plant emissions according to their electricity consumption[3-4]. Other pollutants such as sulfur and particulates are also significantly reduced. In view of the advantages of electric vehicles, the development strategy of new electric vehicles has been promulgated and implemented by the countries and regions represented by the United States, Japan and the European Union[5]. China's Ministry of Science and Technology announced the special plan for the development of electric vehicle technology. The development of electric vehicles is an important way to improve the competitiveness of the automotive industry, ensure energy security and develop low carbon economy[6-8]. In the coming years, it will be a strategic opportunity for research and industrialization of electric vehicles. The national programme for climate change also announced measures to encourage the production and consumption of hybrid electric vehicles and pure electric vehicles[9].

However, due to the short mileage of electric vehicles and lagging behind in charging facilities, the promotion and use of electric vehicles have been severely restricted[10]. To improve the endurance of the electric vehicle by its own technical research and development, it is of great significance to improve the electric vehicle's mileage by planning energy saving path and improving driver's driving control mode, which is very important for the promotion of electric vehicle at this stage.

\section{Energy Consumption Data Collection of Electric Vehicles}

The actual driving mode of outdoor roads can provide a more realistic operation environment. The test results are more accurate, but the application cost is high and the adjustment is more difficult. At the 
same time, a lot of data are needed to cover all kinds of driving states of vehicles. The basic parameters of the electric vehicle used in this article are shown in Table 1.

Table 1 Vehicle performance

\begin{tabular}{|c|c|c|}
\hline \multirow{2}{*}{ Dimension parameter } & Vehicle size $(\mathrm{mm})$ & $4130 \times 1750 \times 1690$ \\
\cline { 2 - 3 } & Wheelbase $(\mathrm{mm})$ & 2620 \\
\hline \multirow{3}{*}{ Weight parameter } & curb weight $(\mathrm{kg})$ & 1580 \\
\cline { 2 - 3 } & Front axle load $(\mathrm{kg})$ & 805 \\
\cline { 2 - 3 } & Rear axle load $(\mathrm{kg})$ & 700 \\
\hline \multirow{4}{*}{ Dynamic performance index } & Maximum speed $(\mathrm{km} / \mathrm{h})$ & $>100$ \\
\cline { 2 - 3 } & Acceleration time $(0-50 \mathrm{~km} / \mathrm{h})$ & $<10$ \\
\cline { 2 - 3 } & Acceleration time $(50-100 \mathrm{~km} / \mathrm{h})$ & $<8$ \\
\cline { 2 - 3 } & Maximum climbing degree & $>20 \%$ \\
\cline { 2 - 3 } & Maximum reversing speed & $12 \mathrm{~km} / \mathrm{h}$ \\
\hline Economic performance index & Driving mileage $(\mathrm{km})$ & $>120$ \\
\hline Battery parameters & Capacity $(\mathrm{Ah})$ & 300 \\
\cline { 2 - 3 } & Voltage $(\mathrm{V})$ & \\
\hline
\end{tabular}

The data collected is shown in Table 2 .

Table 2 The collecting raw data

\begin{tabular}{|c|c|c|c|c|}
\hline Serial number & Accumulative time $(\mathrm{s})$ & Speed $(\mathrm{km} / \mathrm{h})$ & Voltage $(\mathrm{V})$ & Current (A) \\
\hline 0 & 0 & 0 & 380.2 & 1.6 \\
\hline 1 & 0.1 & 0 & 380.0 & 1.53 \\
\hline$\ldots$ & $\ldots$ & $\ldots$ & $\ldots$ & $\ldots$ \\
\hline 1000 & 100 & 10 & 373.2 & 5.05 \\
\hline
\end{tabular}

\section{Energy Consumption Model}

Suppose an integral operator is $\mathrm{K}$. Let $\mathrm{K}$ be a compact operator from Banach space $V$ to $V_{n}$. Thus equation (1) can be written as an operator scheme expression,

$$
\mathrm{K} u=f
$$

The steps of model design are as follows.

STEP1. Choose a sequence $V_{n} \subset V(n \geq 1)$, where the dimension of $V_{n}$ is $N$, in a finite dimensional subspace.

STEP2. Choose Haar wavelet basis $\left\{h_{i}\right\}_{i=1}^{N}$ in $V_{n}$; suppose $u_{n} \in V_{n}$ is the numerical value of equation (9), and then use $u_{n}$ to approach $u . u_{n}(x)$ can be expand into equation (2), where $c_{i}=\left\langle u_{n}(x), h_{i}(x)\right\rangle$ holds and $\left\{c_{i}\right\}_{i=1}^{N}$ is unknown.

$$
u(x) \approx u_{n}(x)=\sum_{i=1}^{N} c_{i} h_{i}(x)
$$

STEP3. Use the traditional Galerkin method. Equation (3) is obtained by substituting equation (2) into integral equation (1). When the remainder term $r_{n}$ in equation (3) is zero, $u_{n}(x)=u(x)$ is obtained. To conveniently get a more accurate numerical solution, $r_{n}(x)$ needs to be as close to zero as possible.

$$
r_{n}(x)=\sum_{i=1}^{N} c_{i} \frac{1}{\Gamma(\alpha)} \int_{0}^{1} k(x, t)(x-t)^{\alpha-1} h_{i}(t) d t-f(x)=\mathrm{K} u_{n}(x)-f(x)
$$

STEP4. Suppose $V=L^{2}[0,1]$, and let $N=2^{J}$ be the wavelet resolution; to meet the requirement of Galerkin method, let $\left\langle r_{n}, h_{i}\right\rangle=0$, where $i=1,2, \cdots, N$; and then with equation (3), a system of linear equations, i.e. equation (4), can be obtained

$$
\sum_{i=1}^{N} c_{i}\left\langle\mathrm{~K} h_{i}, h_{j}\right\rangle=\left\langle f, h_{j}\right\rangle, j=1,2, \cdots, N
$$




\section{Simulation of Energy Consumption in the Model}

In order to test the property of data and realize optimum energy consumption in model, the stimulation of experiment is carried out. Having a model stimulation by Matlab needs to store vehicle performance data and schedule task. Time internal of data sample is $0.1 \mathrm{~s}$ and central time of travelling is $t_{0}=15 \mathrm{~s}$. According to stimulation environment and parameter setting mentioned above, it can gain the wave forms in time domain of energy consumption data through optimizing data and sampling data.

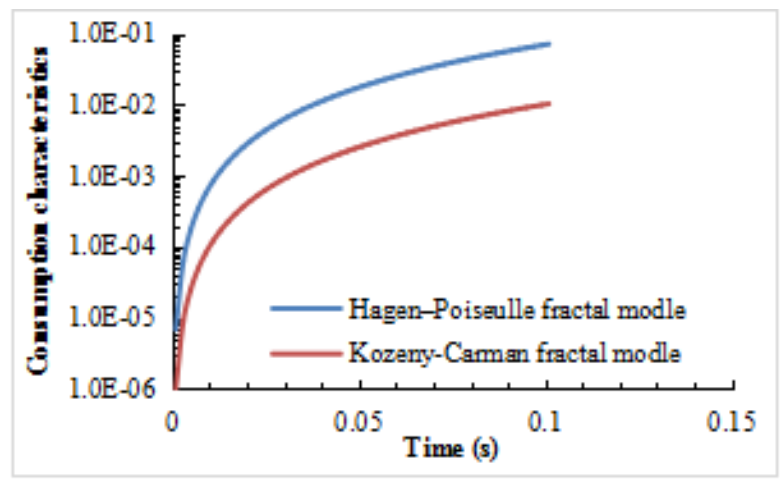

Figure 1. he relationship between the energy consumption characteristics and time from 0 to 0.15

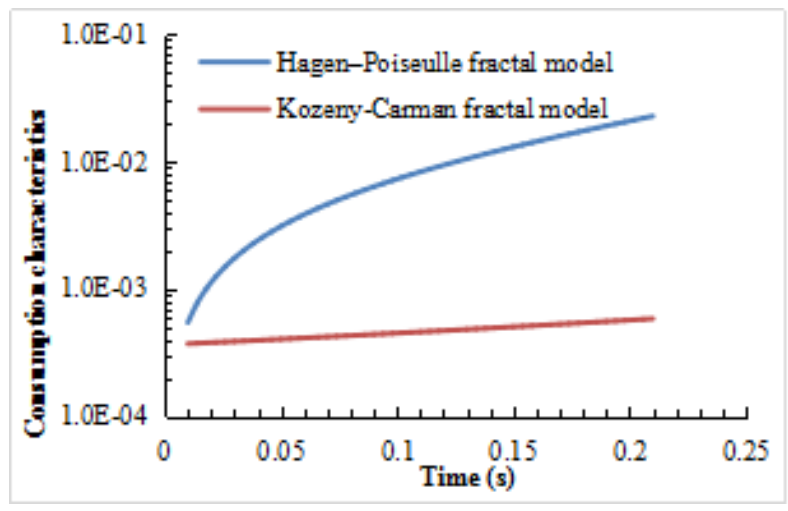

Figure 2. The relationship between the energy consumption characteristics and time from 0 to 0.25

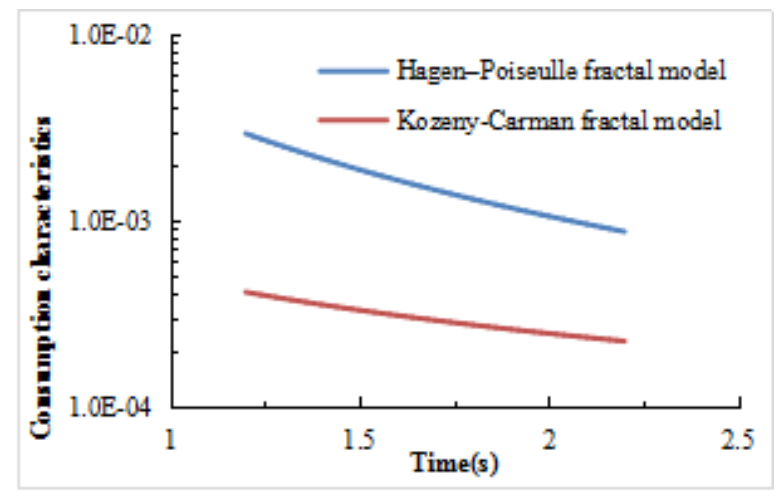

Figure 3. The relationship between the energy consumption characteristics and time from 1 to 2.5

The results show that the way of slow acceleration is taken to save more energy in the acceleration starting and accelerating process of moving scene. the way of slow deceleration is taken to save more energy consumption in braking and deceleration scene during driving. In vehicle route $500 \mathrm{~m}$ scene, the hook speed and deceleration can be taken in energy saving uniform running at different speeds. In order to save energy, the experimental vehicle should try to follow the economic driving speed of about $40 \mathrm{~km} / \mathrm{h}$. 


\section{Summary}

Combined with the research results related to the establishment of energy consumption model of ideas, this paper determines the energy consumption of electric vehicle model by using the running data. At the same time, the acceleration will be divided into acceleration and deceleration and hook speed condition according to the running condition for the detailed description of the relationship between energy consumption rate and the electric vehicle speed and acceleration, and the energy consumption models are established. The energy consumption rate showed a rising trend with increasing acceleration at the same speed. From the overall analysis, the peak energy consumption rate in speed and acceleration are part of the larger, and energy consumption rate has remained low when the acceleration is less than or equal to zero parts.

\section{References}

[1] A Bandyopadhyay and Majumdar. Network Flow Simulation of Fluid Transients in Rocket Propulsion Systems, Journal of Propulsion \& Power, 30 (2015), 1646-1653.

[2] A Lamghari and R Dimitrakopoulos. Network-flow based algorithms for scheduling production in multi-processor open-pit mines accounting for metal uncertainty, European Journal of Operational Research, 250 (2016), 273-290.

[3] BA Akinnuwesi, FME Uzoka, SO Olabiyisi and EO Omidiora. A Framework for User-centric Model for Evaluating the Performance of Distributed Software System Architecture, Expert Systems with Applications, 39(2016), 9323-9339.

[4] C Ley-Bosch, I Alonso-González and D Sánchez-Rodríguez. Evaluation of the Effects of Hidden Node Problems in IEEE 802.15.7 Uplink Performance, Sensors, 16 (2016), 216-227.

[5] CV Karsten, D Pisinger and S Ropke. The time constrained multi-commodity network flow problem and its application to liner shipping network design, Transportation Research Part E Logistics \& Transportation Review,76(2016), 122-138.

[6] F Pierazzi, S Casolari and M Colajanni. Exploratory security analytics for anomaly detection, Computers \& Security, 56(2016), 28-49.

[7] H Chaudhary, DK Yadav, R Bhatnagar and U Chandrasekhar. MapReduce based Frequent Itemset Mining Algorithm on Stream Data, Communication Technologies, 52(2015), 598-603.

[8] N Medvidovic and RN Taylor. A Classification and Comparison Framework for Software Architecture Description Languages, IEEE Transactions on Software Engineering, 26(2016), 70-93.

[9] Y Zhao, ZH Guo and JM Yan. Vibration Signal Analysis and Fault Diagnosis of Bogies of the High-speed Train based on Deep Neural Networks, Journal of Vibroengineering, 19(2017), 2456-2474.

[10]Erdogan, S., Ilçi, V. and Soysal, O. M. A model suggestion for the determination of the traffic accident hotspots on the turkish highway road network: A pilot study, British Journal of Haematology, 21(2015), 69-188. 